\title{
Doping- and size-dependent suppression of tunneling in carbon nanotubes
}

\author{
S. Bellucci, ${ }^{1}$ J. González, ${ }^{2}$ and P. Onorato ${ }^{1,3}$ \\ ${ }^{1}$ INFN, Laboratori Nazionali di Frascati, P.O. Box 13, 00044 Frascati, Italy \\ ${ }^{2}$ Instituto de Estructura de la Materia, Consejo Superior de Investigaciones Científicas, Serrano 123, 28006 Madrid, Spain \\ ${ }^{3}$ Dipartimento di Scienze Fisiche, Università degli Studi di Napoli "Federico II," Via Cintia, I-80126 Napoli, Italy
}

(Received 14 May 2003; revised manuscript received 2 October 2003; published 11 February 2004)

\begin{abstract}
We study the effect of doping in the suppression of tunneling observed in multi-walled nanotubes, incorporating as well the influence of the finite dimensions of the system. A scaling approach allows us to encompass the different values of the critical exponent $\alpha$ measured for the tunneling density of states in carbon nanotubes. We predict that further reduction of $\alpha$ should be observed in multi-walled nanotubes with a sizable amount of doping. In the case of nanotubes with a very large radius, we find a pronounced crossover between a highenergy regime with persistent quasiparticles and a low-energy regime with the properties of a one-dimensional conductor.
\end{abstract}

DOI: 10.1103/PhysRevB.69.085404

PACS number(s): 71.10.Pm, 71.20.Tx, 72.80.Rj

In recent years there has been much interest in the investigation of the electronic properties of carbon nanotubes (CN). ${ }^{1}$ The reduced dimensionality of these systems leads to the appearance of unconventional effects, such as a suppression of the tunneling conductance at low energy scales. This has been interpreted as a signature of the so-called Luttinger liquid behavior, ${ }^{2,3}$ characterized by the absence of electron quasiparticles in the spectrum. Evidence of a power-law behavior in the tunneling density of states at low energies has been obtained from measurements in ropes, ${ }^{4}$ individual single-walled nanotubes (SWNT) ${ }^{5}$ and multi-walled nanotubes (MWNT). ${ }^{6}$

In the Luttinger liquid picture, critical exponents of observables like the density of states are not universal and depend on the interaction strength. In the case of $\mathrm{CN}$, this refers to the ubiquitous Coulomb interaction. However, a precise determination of the interaction strength is precluded by the fact that the electron-electron interaction is actually long-ranged, and it can be assimilated to a coupling constant, only after introducing a suitable infrared cutoff in the singular expression of the one-dimensional (1D) Coulomb potential. ${ }^{7,8}$

Furthermore, the shape of $\mathrm{CN}$ becomes relevant in setting the strength of the electron correlations. In the case of MWNT, measurements of the conductance refer usually to the outer layer, whose electronic properties are influenced by the interaction with inner metallic cylinders. ${ }^{9}$ Also, MWNT use to be significantly doped, which leads to the presence of a large number of subbands at the Fermi level. ${ }^{10}$ The contribution of a large number of modes at low energies has then an appreciable impact on the enhancement of observables like the tunneling density of states.

The purpose of the present paper is to study the combined effect of the finite length of the electron system, on the one hand, and of the number of subbands at low energies, on the other hand, in the Luttinger liquid description of $\mathrm{CN}$. There have been precedent studies devoted to understanding the effects of coupling a large number of subbands in onedimensional electron systems. ${ }^{11}$ The main difference introduced by our analysis is that we focus on the case of the long-range Coulomb interaction, which is the relevant in- stance for $\mathrm{CN}$ under common experimental conditions. Then, the main effects of the interaction come from scattering processes with low momentum transfer, in which the repulsive interaction is enhanced. From a 1D point of view, the Coulomb potential has a logarithmic dependence on the momentum ${ }^{2}$

$$
V_{C}(k) \approx(1 / 2 \pi) \ln \left(k_{c} / k\right)
$$

where $k_{c}$ is a short-distance cutoff, of the order of the inverse of the diameter $d$ in the case of the $\mathrm{CN}$. The momentum transfer $k$ is only limited in the infrared by the inverse of the nanotube length $L$. This means that, for typical experimental samples which are about one micron long, the scattering processes with generic momentum transfer have a strength about one order of magnitude below that of the forwardscattering processes. We will then consider the regime where $L$ is several orders of magnitude greater than $d$, in which backscattering and Cooper-pair processes can be safely disregarded in favor of those with forward-scattering probing the singular character of the repulsive interaction.

We will actually show that a precise treatment of the longrange character of the Coulomb interaction allows one to account for the suppression of the tunneling density of states observed in the MWNT. It has been found that the exponents for the low-energy power-law behavior measured experimentally are relatively large, compared to the results obtained in a conventional Luttinger liquid description. ${ }^{6}$ This has led to the proposal that a nonconventional Coulomb blockade effect including disorder must be responsible for the lowenergy behavior observed in the experiments. ${ }^{12}$ The results from our study imply that the long-range character of the Coulomb interaction has to be taken into account for a complete description of the MWNT, as the consequent enhancement of the forward-scattering processes provides a complementary way of understanding the relatively large suppression of the tunneling density of states.

With this aim, we adopt a renormalization group (RG) approach, which is well suited to obtain the energy dependence of quantities like the quasiparticle weight. ${ }^{13}$ In the representation (1) of the potential, the logarithmic depen- 
dence on $k$ leads to an imperfect scaling behavior, making the use of the RG approach not quite appropriate. In order to cure the infrared singularities arising from the long-range interaction, we implement a dimensional regularization of the theory, writing formally all bare quantities slightly away from dimension $D=1 .^{14,15}$ This formal artifact has a real physical meaning since, as we are going to see, the deviation $D-1$ yields a measure of the finite size of the system, with the limit $D \rightarrow 1$ corresponding to the case of increasingly long nanotubes.

The proposed regularization of the long-range interaction provides a sensible description of the low-energy properties of the electron system, as the interacting theory remains scale invariant, up to logarithmic corrections, at any dimension above $D=1$. Let us consider the case of a generic twocomponent electron field $\Psi(\mathbf{r})$ with linear dispersion and subject to the long-range Coulomb interaction. We can write the action at general dimension $D$ in the form

$$
\begin{aligned}
S= & \int d t \int d^{D} r \Psi^{+}(\mathbf{r})\left(i \partial_{t}+i v_{F} \boldsymbol{\sigma} \cdot \nabla\right) \Psi(\mathbf{r}) \\
& -e^{2} \int d t \int d^{D} r \int d^{D} r^{\prime} \Psi^{+}(\mathbf{r}) \\
& \times \Psi(\mathbf{r}) \frac{1}{\left|\mathbf{r}-\mathbf{r}^{\prime}\right|} \Psi^{+}\left(\mathbf{r}^{\prime}\right) \Psi\left(\mathbf{r}^{\prime}\right),
\end{aligned}
$$

where the $\sigma_{i}$ matrices are defined formally by $\left\{\sigma_{i}, \sigma_{j}\right\}$ $=2 \delta_{i j}$. Under a change in the scale $\varepsilon$ of the energy variables in the model,

$$
\varepsilon \rightarrow s \varepsilon,
$$

we must have accordingly

$$
t \rightarrow s^{-1} t .
$$

The requirement of scale invariance for the kinetic term in Eq. (2) dictates the transformation rules

$$
\begin{gathered}
\mathbf{r} \rightarrow s^{-1} \mathbf{r}, \\
\Psi(\mathbf{r}) \rightarrow s^{D / 2} \Psi(\mathbf{r}) .
\end{gathered}
$$

The important point is that the interaction term in the action (2) remains scale invariant under the scaling transformation (3)-(6). It can be checked that additional interactions mediated by a local potential or with higher content of electron fields lead to terms scaling with positive powers of the $s$ factor, therefore being irrelevant in the low-energy limit $s$ $\rightarrow 0$. The same applies to terms accounting for higher-order corrections to the linear dispersion relation. This means that the model given at the classical level by Eq. (2) is a good starting point to study the low-energy properties of the theory at the quantum level, since it is not going to be perturbed by the appearance of additional interactions that may grow large in the low-energy limit.

The Coulomb potential $1 /|\mathbf{r}|$ can be represented in three spatial dimensions as the Fourier transform of the propagator $1 / \mathbf{k}^{2}$

$$
\frac{1}{|\mathbf{r}|}=\int \frac{d^{3} k}{(2 \pi)^{3}} e^{i \mathbf{k} \cdot \mathbf{r}} \frac{1}{\mathbf{k}^{2}} .
$$

If the interaction is projected onto one spatial dimension, by integrating for instance the modes in the two transverse dimensions, then the Fourier transform has the usual logarithmic dependence on the momentum shown in Eq. (1), $k_{c}$ being the memory that the system keeps of the finite transversal size. We choose instead to integrate formally a number 3 $-D$ of dimensions, so that the long-range potential gets the representation

$$
\frac{1}{|x|}=\int \frac{d^{D} k}{(2 \pi)^{D}} e^{i k x} \frac{c(D)}{|\mathbf{k}|^{D-1}},
$$

where $c(D)=\Gamma((D-1) / 2) /(2 \sqrt{\pi})^{3-D}$. In the limit $D \rightarrow 1$, the expression of the potential in momentum space becomes

$$
\frac{c(D)}{|\mathbf{k}|^{D-1}} \approx \frac{1}{2 \pi}\left(\frac{1}{D-1}-\ln (|\mathbf{k}|)+\cdots\right) .
$$

Expression (9) shows, after comparison with Eq. (1), that $1 /(D-1)$ corresponds actually to the logarithm of the shortdistance cutoff $k_{c}$. It may seem at first glance that this relation is not precisely defined, since $k_{c}$ is a dimensionful parameter. This ambiguity is however resolved in the framework of the RG, in which one predicts the behavior of the observables upon changes in the scale. As long as the momenta are scaled down to zero in order to reach the lowenergy limit of the theory, the quantity $1 /(D-1)$ stands for the logarithm of the ratio between the highest and the lowest momentum attained in the scaling procedure. The latter is constrained by the length of the electron system, which acts as an infrared cutoff. Thus, the dimensionless quantity $1 /(D-1)$ has to be traded for the logarithm of the length $L$ of the system, measured in units of the finite nanotube diameter $d$. This argument makes clear that such an equivalence has asymptotic character, and that it must hold provided that $L \gg d>0$.

In order to proceed with the RG program, we write then the Hamiltonian for the linear branches of $\mathrm{CN}$ in the form

$$
\begin{aligned}
H= & v_{F} \sum_{\alpha \sigma} \int_{0}^{E_{c}{ }^{\prime v_{F}}} d p|\mathbf{p}|^{D-1} \int \frac{d \Omega}{(2 \pi)^{D}} \Psi_{\alpha \sigma}^{+}(\mathbf{p}) \boldsymbol{\sigma} \cdot \mathbf{p} \Psi_{\alpha \sigma}(\mathbf{p}) \\
& +e^{2} \int_{0}^{E_{c}{ }^{\prime v_{F}}} d p|\mathbf{p}|^{D-1} \int \frac{d \Omega}{(2 \pi)^{D}} \rho(\mathbf{p}) \frac{c(D)}{|\mathbf{p}|^{D-1}} \rho(-\mathbf{p}),
\end{aligned}
$$

where $\rho(\mathbf{p})$ are density operators made of the electron fields $\Psi_{\alpha \sigma}(\mathbf{p})$, with $\alpha$ labeling the Fermi point and $\sigma$ the spin projection. The sum in Eq. (10) runs then over the usual four modes present in a single-walled nanotube, but it may include also the contribution from a large number of subbands in the case of a doped multi-walled nanotube.

We focus on the scaling properties of the model as the energy cutoff $E_{c}$ is lowered, when a large number $N$ of subbands contribute to the electronic properties down to the 
Fermi level. Each subband is labeled with a different quantum number, which corresponds to the momentum in the dimension around the nanotube. For this reason, the dominant processes are those where each scattered electron remains in the same linear branch. The main effect of the interaction is to dress the bare electron propagator with the polarization of the $N$ different subbands given by

$$
\Pi\left(\mathbf{k}, \omega_{k}\right)=2 N b(D) \frac{v_{F}^{2-D} \mathbf{k}^{2}}{\left|v_{F}^{2} \mathbf{k}^{2}-\omega_{k}^{2}\right|^{(3-D) / 2}},
$$

where $\quad b(D)=(2 / \sqrt{\pi})\left[\Gamma((D+1) / 2)^{2} \Gamma((3-D) / 2) /\right.$ $\left.(2 \sqrt{\pi})^{D} \Gamma(D+1)\right] .{ }^{16}$ The polarization (11) is the analytic continuation of the known result for two linear branches with opposite chirality, which we take away from $D=1$ in order to carry out a consistent regularization of the Coulomb interaction. After dressing the interaction with the polarization (11), the electron self-energy is given by the expression

$$
\begin{aligned}
\Sigma\left(\mathbf{k}, \omega_{k}\right)= & -e^{2} \int_{0}^{E_{c}{ }^{\prime v_{F}}} d p|\mathbf{p}|^{D-1} \int \frac{d \Omega}{(2 \pi)^{D}} \int \frac{d \omega_{p}}{2 \pi} \\
& \times G\left(\mathbf{k}-\mathbf{p}, \omega_{k}-\omega_{p}\right) \frac{-i}{\frac{|\mathbf{p}|^{D-1}}{c(D)}+e^{2} \Pi\left(\mathbf{p}, \omega_{p}\right)} .
\end{aligned}
$$

The low-energy properties of the theory are investigated by taking the limit $E_{c} \rightarrow 0$, where the self-energy $\Sigma$ turns out to have terms linear in $\omega_{k}$ and $\mathbf{k}$ that depend logarithmically on the cutoff. This is the signal that the scale of the electron wave function and the Fermi velocity $v_{F}$ are renormalized at low energies. Then one has to assume that the bare electron field, for which the correlators are computed, has a dependence on the cutoff $E_{c}$ of the form

$$
\Psi_{\text {bare }}\left(E_{c}\right)=Z^{1 / 2}\left(E_{c}\right) \Psi,
$$

where $\Psi$ is the cutoff-independent electron field. The divergent contributions to the electron propagator read

$$
\begin{aligned}
\frac{1}{G}= & \frac{1}{G_{0}}-\Sigma \approx Z^{-1}\left(\omega_{k}-v_{F} \boldsymbol{\sigma} \cdot \mathbf{k}\right) \\
& -Z^{-1} \frac{f(D)}{2 N} \sum_{n=0}^{\infty}(-1)^{n} g^{n+1}\left(\frac{n(3-D)}{n(3-D)+2} \omega_{k}\right. \\
& \left.+\left(1-\frac{2}{D} \frac{n(3-D)+1}{n(3-D)+2}\right) v_{F} \boldsymbol{\sigma} \cdot \mathbf{k}\right) h_{n}(D) \log \left(E_{c}\right),
\end{aligned}
$$

where

$$
\begin{gathered}
g=2 N b(D) c(D) e^{2} / v_{F}, \quad h_{n}(D)=\frac{\Gamma(n(3-D) / 2+1 / 2)}{\Gamma(n(3-D) / 2+1)}, \\
f(D)=\frac{1}{2^{D} \pi^{(D+1) / 2} \Gamma(D / 2) b(D)} .
\end{gathered}
$$

The usual RG argument is that the renormalized propagator $G$ must be a finite quantity, so that the divergent dependencies on the cutoff $E_{c}$ have to be reabsorbed in the scale of the wave function $Z^{1 / 2}$ and the Fermi velocity $v_{F} \cdot{ }^{17}$ Under a differential variation of $E_{c}, Z^{1 / 2}$ is renormalized according to

$$
\begin{aligned}
E_{c} \frac{d}{d E_{c}} \log Z\left(E_{c}\right)= & -\frac{f(D)}{2 N} \sum_{n=0}^{\infty}(-1)^{n} g^{n+1} \\
& \times \frac{n(3-D)}{n(3-D)+2} h_{n}(D) .
\end{aligned}
$$

The renormalization of $v_{F}$ can be translated into that of the effective coupling $g=2 N b(D) c(D) e^{2} / v_{F}$, since the electron charge $e$ is not renormalized in our model. The RG equation for $g$ becomes

$$
\begin{aligned}
E_{c} \frac{d}{d E_{c}} g\left(E_{c}\right)= & \frac{f(D)}{2 N} \frac{2(D-1)}{D} g^{2} \sum_{n=0}^{\infty}(-g)^{n} \\
& \times\left(\frac{(3-D) n+1}{(3-D) n+2}\right) h_{n}(D) .
\end{aligned}
$$

We are now in a position to study the influence of the long-range Coulomb interaction in the limit $D \rightarrow 1$. For this purpose, we start by considering the RG equation (16). The series shown on the right hand side (rhs) can be summed up at $D=1$, the flow equation taking then the following form in the neighborhood of that point:

$$
E_{c} \frac{d}{d E_{c}} g\left(E_{c}\right) \approx \frac{1}{2 N}(D-1) g\left(1-\frac{1}{\sqrt{1+g}}\right) .
$$

It follows from Eq. (17) that, at $D=1$, there is formally a line of fixed points covering all values of the interaction strength. However, we still have to take into account that the effective coupling $g(D)$ becomes singular in the limit $D$ $\rightarrow 1$. We can shuffle this divergence into the initial value of the coupling, $g_{0}(D)$, which turns out to have near $D=1$ the asymptotic behavior

$$
g_{0}(D) \approx N \frac{e^{2}}{\pi^{2} v_{F}} \frac{1}{D-1} .
$$

Then, by matching the behavior of $g_{0}(D)$ with that of Eq. (17), we observe that the $D-1$ factor in the rhs of the RG equation is not completely canceled out in the limit $D \rightarrow 1$. This shows in a rigorous way that the bare 1D long-range interaction is at a RG fixed point for arbitrary values of the interaction strength.

We next consider the RG equation (15) for the electron wave function scale in the limit $D \rightarrow 1$. The series on the rhs can be also summed up at $D=1$, with the result that the scaling equation in that limit reads

$$
E_{c} \frac{d}{d E_{c}} \log Z\left(E_{c}\right) \approx \frac{1}{4 N}\left(\sqrt{1+g}+\frac{1}{\sqrt{1+g}}-2\right) .
$$


The function on the rhs of Eq. (19) coincides with the anomalous electron dimension found in the exact solution of the Luttinger model. ${ }^{18,19}$ This ensures that the RG approach is a sensible way to obtain the low-energy properties of the model. We have to bear in mind that our description introduces however two important differences with respect to the usual treatment of 1D interacting electrons. On the one hand, the slight deviation of the model from $D=1$ allows one to control the approach to the bare long-range interaction as the length of the system is increased. On the other hand, we have also incorporated the effect of the number $N$ of subbands that contribute at low energies, in order to account for the influence of doping in MWNT.

With the RG approach we can face two different experimental conditions, depending on the magnitude of the typical energy scale involved in the measurement process, as compared to the spacing between subbands in the nanotube sample. When the latter is larger than the temperature or bias voltage applied to the sample, we are in a situation where the number of subbands $N$ can be taken as constant along the RG flow. Otherwise, for large enough temperature or bias voltage, the number of subbands that contribute at the scale of the high-energy cutoff is a decreasing variable as $E_{c} \rightarrow 0$. We deal separately with the two instances in what follows.

$R G$ approach with a constant number $N$ of subbands. In transport experiments, the typical scale of temperature or bias voltage lies usually below the scale of the spacing between subbands. This has been so, even in the measurements made in MWNT, where the spacing corresponding to a typical diameter $d \approx 17 \mathrm{~nm}$ is as small as $\hbar v_{F} / d \approx 29 \mathrm{meV}$. In these conditions, the only subbands that contribute to the properties measured experimentally are those crossing the Fermi level.

We apply then Eqs. (18) and (19) to confront the experimental results on the tunneling density of states gathered from different nanotube samples. Starting with the measurements made in SWNT, we take a number of subbands $N$ $=2$ in the equations and adjust the deviation from $D=1$ in accordance to the length of the experimental sample. Following the argument below Eq. (9), we use the correspondence $1 /(D-1) \approx \ln (L / d), L$ being the nanotube length and $d$ the nanotube diameter. A suitable choice corresponding to the experiments reported in Ref. 5 is $L / d \sim 10^{3}$, which gives in turn $D \approx 1.14$.

The behavior of the scale $Z^{1 / 2}$ of the electron wave function can be used to establish the phase in which the electron system falls at low energies. A finite value of $Z\left(E_{c}\right)$ in the limit $E_{c} \rightarrow 0$ is the signature of the persistence of electron quasiparticles near the Fermi level. The power-law dependence of $Z\left(E_{c}\right)$ on $E_{c}$ points instead at the incoherent propagation of fermion-like excitations and the onset of Luttinger liquid behavior. For the values of $D$ which are estimated relevant for the experiments, it can be checked that the coupling $g\left(E_{c}\right)$ obtained from Eq. (17) is far away from the region of attraction of the infrared free fixed point. It has been actually shown in Ref. 14 that, for values of $D$ below 1.2 , the dependence of $Z\left(E_{c}\right)$ cannot be distinguished from a power-law behavior

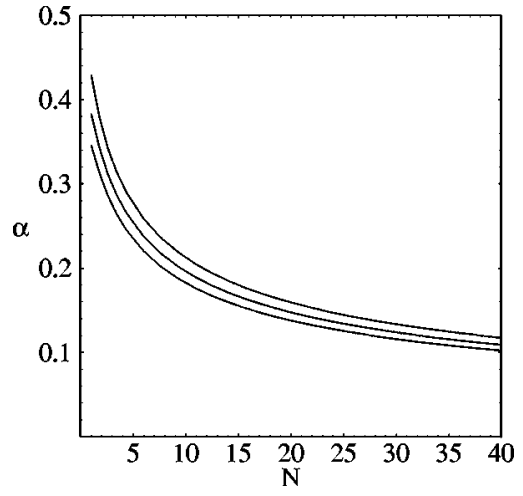

FIG. 1. Estimates of the exponent $\alpha$ from the rhs of Eq. (19), for $e^{2} / \pi^{2} v_{F} \approx 1.5$. The different curves correspond, from top to bottom, to $D=1.14,1.16$, and 1.18 .

$$
Z\left(E_{c}\right) \sim E_{c}^{\alpha}
$$

when the scaling is performed down to energies which are up to four orders of magnitude below the characteristic highenergy scale of the model. Thus, the crossover to a stable behavior of $Z\left(E_{c}\right)$ turns out to happen at such small energies that it is not observable in practice. For the typical scales at which the experiments are performed (down to energies of the order of a few meV), one can safely estimate the exponent $\alpha$ controlling the power-law behavior of the density of states from the rhs of Eq. (19).

The measurements of the tunneling density of states in SWNT have shown a power-law dependence on energy, with values of the critical exponent $\alpha$ accumulating around $\approx 0.35$. ${ }^{4,5}$ We have represented in Fig. 1 the estimates obtained from the rhs of Eq. (19), for small values of $D-1$. The values of $\alpha$ have a smooth dependence on the length $L$ of the system and fall around $\alpha \approx 0.35$ for $N=2$ and $D$ $\approx 1.14$, with a reasonable choice of the coupling $e^{2} / \pi^{2} v_{F}$ $\approx 1.5$.

Our results also show an overall agreement with the exponents measured in MWNT. It has been noticed that such systems are significantly doped, so that a large number of subbands are found at the Fermi level. The experimental conditions refer to a situation where $N \approx 5-10$ (without taking into account the spin degeneracy). It has been reported that the values of the critical exponent $\alpha$ measured in 11 different samples range from 0.24 to $0.37 .^{6}$ This variation can be accounted for within our RG approach by assuming that the number of subbands used in the renormalization may shift from $N=2$ to $N=10$. Part of the drift observed in the critical exponent may also be due to the smaller aspect ratio of MWNT, although this fact is difficult to assess given the lack of information about the total length of the experimental samples.

We show then that the suppression of tunneling in MWNT can be softened by increasing the doping level. Our results may also be relevant in general for nanotubes of large radius where there are a large number of subbands crossing the Fermi level. These instances can be considered as midway in the process of making contact with the physical properties of a graphene sheet. This requires taking systems with larger 


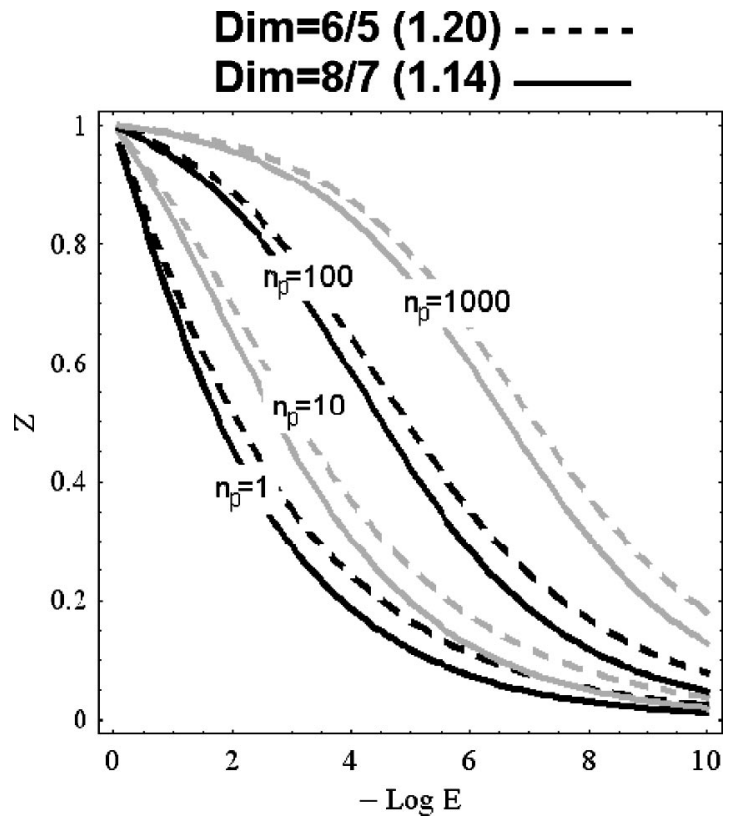

FIG. 2. (Color online) Energy dependence of the quasiparticle weight $Z$ at dimensions $6 / 5$ and $8 / 7$, for different values of $n_{p}$.

transverse size, which in turn may lead to a situation where the spacing between subbands is smaller than the typical energy scale in the experimental measurements. One then has to change slightly the computational scheme, as discussed in the following.

$R G$ approach with a cutoff-dependent number of subbands $N\left(E_{c}\right)$. In samples of a very large diameter, one may envisage conditions where the temperature or the bias voltage are much larger than the subband spacing in the nanotube. The RG approach can be still implemented, but taking into account that the number of subbands contributing in the partial integration of modes at energy $E_{c}$ depends on the value of the high-energy cutoff. Let us suppose for simplicity that the system is at half-filling, with the typical structure of two subbands crossing at the Fermi level. For not too large energies, the number of subbands crossing the energy level $\varepsilon$ then has a linear dependence on $\varepsilon, N(\varepsilon)=N_{0}+n_{p} \varepsilon$, where $N_{0}=2$ is the number of subbands at the Fermi level and $n_{p}$ represents the number of subbands, in a unit of energy normalized with respect to $N_{0}$.

We obtain the scaling of the quasiparticle weight $Z$ and the different observables by imposing a dependence of $N$ on the cutoff $E_{c}$ according to the above-mentioned formula. This description yields a sensible prediction for experiments where the average effect of a large number of subbands is measured. The dependence of the quasiparticle weight $Z$ on energy as the cutoff is sent toward the Fermi level is represented in Fig. 2, for different values of $n_{p}$. In the present instance, the dominant contribution to the power-law behavior of the tunneling density of states comes from the number of subbands varying with the energy scale. This sets the value of $\alpha$ close to 1 from the start, which is the natural way of recovering the characteristic linear density of states of a graphite layer from our 1D point of view.

We observe from the results in Fig. 2 that the quasiparticle weight $Z$ tends to have a flat behavior at high energies for large values of the density of subbands $n_{p}$. This is in contrast to the rapid decrease signaling the typical power-law behavior for small values of $n_{p}$. In the curves for $n_{p}=100$ and $n_{p}=1000$, we see the existence of a crossover between a regime with persistent quasiparticles and another characteristic of the Luttinger liquid behavior. The physical interpretation is that, for high energies above the crossover scale, the system has similar properties to the $2 \mathrm{D}$ graphene, while one has to look at sufficiently small energy scales (or large length scales) to measure the properties of the $1 \mathrm{D}$ wire.

We have obtained several results that may be checked against future measurements carried out in MWNT and nanotubes of very large radius. We predict that the exponent giving the power-law behavior of the tunneling density of states may suffer a significant reduction upon doping those systems, with the possibility of reaching values as small as 0.1 for $N \approx 40$. We have also shown that, when dealing with nanotubes of very large radius, there is a high-energy regime with persistent electron quasiparticles which has properties closer to two-dimensional graphene than to the Luttinger liquid. We believe such features may be of interest when developing carbon-based devices made of graphene and nanotube structures with different shapes.

This work is partly supported by the Italian Research Ministry, National Interest Program.
${ }^{1}$ A. Bachtold, P. Hadley, T. Nakanishi, and C. Dekker, Science 294, 1317 (2001).

${ }^{2}$ R. Egger and A. O. Gogolin, Phys. Rev. Lett. 79, 5082 (1997); Eur. Phys. J. B 3, 281 (1998).

${ }^{3}$ C. Kane, L. Balents, and M. P. A. Fisher, Phys. Rev. Lett. 79, 5086 (1997).

${ }^{4}$ M. Bockrath, D. H. Cobden, J. Lu, A. G. Rinzler, R. E. Smalley, L. Balents, and P. L. McEuen, Nature (London) 397, 598 (1999).

${ }^{5}$ Z. Yao, H. W. Ch. Postma, L. Balents, and C. Dekker, Nature (London) 402, 273 (1999).

${ }^{6}$ A. Bachtold, M. de Jonge, K. Grove-Rasmussen, P. L. McEuen, M. Buitelaar, and C. Schönenberger, Phys. Rev. Lett. 87, 166801 (2001); A. Bachtold, M. de Jonge, K. Grove-Rasmussen,
P. L. McEuen, M. Buitelaar, and C. Schönenberger, cond-mat/0012262.

${ }^{7}$ R. Egger and H. Grabert, Phys. Rev. Lett. 79, 3463 (1997).

${ }^{8}$ S. Bellucci and J. González, Eur. Phys. J. B 18, 3 (2000).

${ }^{9}$ R. Egger, Phys. Rev. Lett. 83, 5547 (1999).

${ }^{10}$ M. Krüger, M. R. Buitelaar, T. Nussbaumer, C. Schönenberger, and L. Forró, Appl. Phys. Lett. 78, 1291 (2001).

${ }^{11}$ L. Balents and M. P. A. Fisher, Phys. Rev. B 53, 12133 (1996); H. J. Schulz, ibid. 53, R2959 (1996); M. Fabrizio, ibid. 48, 15838 (1993); T. Kimura, K. Kuroki, and H. Aoki, ibid. 54, R9608 (1996); H.-H. Lin, L. Balents, and M. P. A. Fisher, ibid. 56, 6569 (1998).

${ }^{12}$ R. Egger and A. O. Gogolin, Phys. Rev. Lett. 87, 066401 (2001). 
${ }^{13}$ R. Shankar, Rev. Mod. Phys. 66, 129 (1994).

${ }^{14}$ S. Bellucci and J. González, Phys. Rev. B 64, 201106(R) (2001).

${ }^{15}$ The method of analytic continuation in the number of dimensions has been proposed in the case of a short-range interaction by $\mathrm{C}$. Castellani, C. Di Castro, and W. Metzner, Phys. Rev. Lett. 72, 316 (1994).
16 C. Itzykson and B. Zuber, Quantum Field Theory (McGrawHill, New York, 1980).

${ }^{17}$ P.-A. Bares and X. G. Wen, Phys. Rev. B 48, 8636 (1993).

18 V. J. Emery, in Highly Conducting One-Dimensional Solids, edited by J. T. Devreese, R. P. Evrard, and V. E. Van Doren (Plenum, New York, 1979).

${ }^{19}$ J. Sólyom, Adv. Phys. 28, 201 (1979). 